interpreted cautiously. Only a few relatives have been examined, and therefore ascertainment of the correct diagnosis by history alone is difficult. Increased heritability in PD is suggested by the occasional occurrence of PD in large kindreds and in monozygotic twins [4]. We and others have provided evidence that a subgroup of PD patients, particularly those with prominent tremor, have a genetic susceptibility for PD $[1,8]$. This notion contrasts with the conclusion from the twin study, in which increased genetic susceptibility could not be demonstrated [7]. However, it is possible that the patients in the twin study had the postural-instability-gaitdifficulty variety of $\mathrm{PD}$ which, in contrast to the tremor type, may not be associated with a genetically increased risk for PD.

According to Dr Lang and his colleagues, the occurrence of tremor, but not of PD, in first-degree relatives of PD patients is three times that expected. Therefore, it must be inferred that the tremor among the relatives is of the familial essential type. Thus, the $1.3 \%$ incidence of coexisting ET and PD is surprisingly low. Also, the $4.4 \%$ incidence of ET in the dystonic population is much lower than that previously reported [2].

Since the publication of our series, we analyzed the cases of additional patients with typical PD and pre-existing ET. Among 833 typical PD patients and 281 ET patients, we identified 56 with a combination of ET and PD. Thus, $6.7 \%$ of all PD and $19.9 \%$ of all ET patients had the combination syndrome. (This compares to the expected prevalence of $0.41 \%$ for ET and $0.35 \%$ for PD $[4,6]$.) The number of patients with coexisting ET and PD would be higher if Parkinson's patients who later developed ET were included. While it is difficult to diagnose ET in patients with preexisting PD because of the frequent occurrence of action tremor in PD, Parkinson's patients are just as likely, if not more so, to develop ET later in life.

Finally, because our patients are referred from a large geographic area, it is difficult to determine the prevalence rate of ET in Houston. This, of course, points to one limitation of our study, since our patients may be more unique and perhaps do not reflect the clinical and hereditary characteristics of the general population. However, by using a matched population of PD patients, we have attempted to correct for this possible bias. Therefore, our conclusion that some ET patients carry an increased risk for PD is valid. The data presented by Dr Lang and associates provide additional evidence that the presence of PD increases the risk for ET.

\section{Baylor College of Medicine}

Texas Medical Center

Houston, TX 77030

\section{References}

1. Barbeau A, Roy M: Familial subsets in idiopathic Parkinson's disease. Can J Neurol Sci 11:144-150, 1984

2. Couch JR: Dystonia and tremor in spasmodic torticollis. Adv Neurol 14:245-258, 1976

3. Geraghty JJ, Jankovic J, Zetusky WJ: Association between essential tremor and Parkinson's disease. Ann Neurol 17:329-333, 1985

4. Haerer AF, Anderson DW, Schoenberg BS: Prevalence of essential tremor. Results from the Copiah county study. Arch Neurol $39: 750-751,1982$
5. Jankovic J, Reches A: Parkinson's disease in monozygotic twins. Ann Neurol (in press)

6. Schoenberg BS, Anderson DW, Haerer AF: Prevalence of Parkinson's disease in the biracial population of Copiah county, Mississippi. Neurology (Cleveland) 35:841-845, 1985

7. Ward CD, Duvoisin RC, Ince SE, et al: Parkinson's disease in 65 pairs of twins and one set of quadruplets. Neurology (Cleveland) 33:815-834, 1983

8. Zetusky WJ, Jankovic J, Pirozzolo FJ: The heterogeneiry of Parkinson's disease: clinical and prognostic implications. Neurology (Cleveland) 35:522-526, 1985

\section{Fatal Pneumocystis Pneumonia from ACTH Therapy for Infantile Spasms}

Mark G. Goetting, MD

Adrenocorticotropic hormone (ACTH) is generally accepted as the drug of choice for the treatment of infantile spasms. Controversy exists over the dose and duration of treatment. Some advocate prolonged therapy at high doses for 10 or more months [5]. I report a case of fatal Pneumocystis carinit pneumonia as a complication of prolonged ACTH therapy.

When first seen, the patient was a $31 / 2$-month-old male infant with clinical and radiological signs of tuberous sclerosis, developmental delay, infantile spasms, and hypsarrhythmia shown on electroencephalogram. He was treated with 80 units of ACTH in gel intramuscularly every other day and became seizure-free in 4 days. Two months later his seizures recurred. His ACTH was increased to 80 units daily. The seizure frequency declined but he developed mucocutaneous candidiasis that responded poorly to topical therapy. He also became hypertensive. After 3 months, oral prednisone (1 mg per kilogram of body weight per day) was substituted for ACTH, with intent of tapering. Clonazepam was used for seizure control. Two days later the mother thought he was "congested." The next day he was found dead in his crib.

Postmortem examination revealed bilateral severe pneumonia. The lung interstitium, including the alveolar and bronchiolar walls, was filled with lymphocytes and plasma cells. The alveoli were lined by a fibrinous exudate and filled with a frothy exudate containing many Pneumocystis organisms. No other pathogens were isolated.

Pneumocystis carinii pneumonia is exceedingly rare in immunocompetent children beyond the first 3 months of life. Prolonged high-dose corticosteroid use has uniformly caused Pneumocystis pneumonia in rats, but has rarely been associated with this infection in humans $[1,3]$. It is believed that corticosteroids interfere with cellular immunity [1]. The patient described here had no past or family history of immune disease and had no other risk factor for opportunistic infection.

The incidence of infectious complications from ACTH use in infancy is related to dose, if not duration [4]. Two 
cases of hospital-acquired Pneumocystis pneumonia during ACTH therapy have been reported [4]. Both patients were treated for 6 weeks and one received 120 units per day. An informal survey of over 600 cases revealed no other patient with known Pneumorystis pneumonia. However a large majority of the patients were treated for less than 6 weeks. In experimental animals, 2 to 3 months of corticosteroid administration is required for infection to develop.

This case demonstrates that Pneumocystis pneumonia is a potential complication of prolonged ACTH therapy in infants. Not enough data exist to assess the risk-benefit ratio of trimethoprim-sulfamethoxazole prophylaxis during ACTH therapy, although this strategy is justified in leukemia [2].

\section{Section of Pediatric Neurology \\ University of Michigan Medical Center \\ C2028 Box 66}

Ann Arbor, MI 48109

\section{References}

1. Frenkl JK, Good JT, Schultz JA: Latent Pneumocystis infection of rats, relapse and chemotherapy. Lab Invest 15:1559-1577, 1966

2. Hughes WT: Natural mode of acquisition for de novo infection with Pneumocystis carinii. J Infect Dis 145:842-848, 1982

3. Hughes WT, Kuhn S, Chudhary S, et al: Successful chemoprophylaxis for Pneumocystis carinii pneumonitis. N Engl J Med 297:1419-1429, 1977

4. Riikonen R, Donner M: ACTH therapy in infantile spasms: side effects. Arch Dis Child 55:664-672, 1980

5. Singer WD, Rabe EF, Haller JS: The effect of ACTH therapy upon infantile spasms. J Pediatr 96:485-489, 1980

\section{Delayed Postirradiation Lower Motor Neuron Syndrome}

\section{J. Gállego, MD, ${ }^{*}$ G. Delgado, MD, ${ }^{*}$ T. Tuñón, MD, $\dagger$} and J. A. Villanueva, MD*

The syndrome of postirradiation flaccid paralysis with amyotrophy of the lower limbs was described by Greenfield and Stark in 1948 [1]. Because of its low incidence, there is little in the literature about this complication $[1-4,6]$. No postmortem or neuromuscular biopsy studies of this rare side effect of radiotherapy have been published to our knowledge. We studied a patient with this syndrome in whom neuromuscular biopsy strongly suggested a chronic lesion of the spinal motor neuron.

A 34-year-old man was diagnosed in 1970 as having a primary bone lymphoma located in the L 3 vertebral body. He was treated by cobalt beam (4,600 rads in 23 daily fractions of $200 \mathrm{rads}$ each). A year later, another lesion was discovered in the T10 and T11 vertebrae. Both lesions were irradiated ( 5,000 rads in 20 daily fractions of 250 rads each). In 1976 another lesion appeared in the T9 vertebra; a total dosage of 5,000 rads in 20 daily fractions of 250 rads each

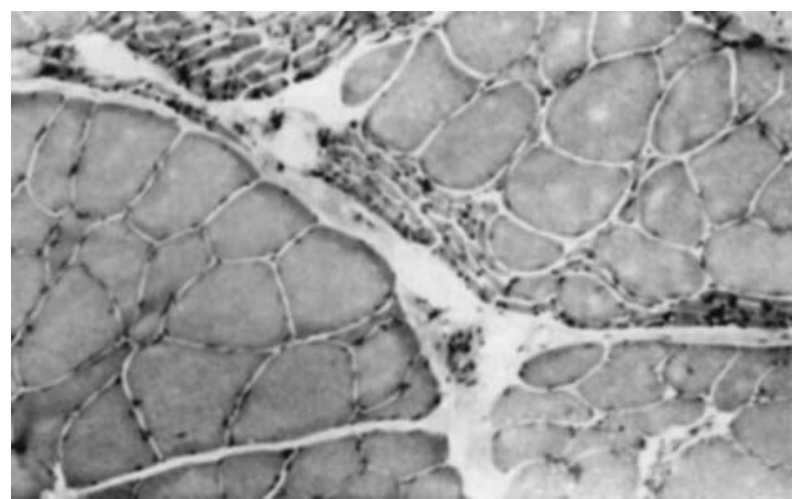

Gastrocnemius muscle. (HEE; $\times 50$ before $43 \%$ reduction.)

was then given. Thirteen months later he began to notice weakness and muscular atrophy in the lower limbs. These symptoms progressed slowly over the following three years.

When we examined the patient in 1982 , his condition was stable subjectively. Neurological examination revealed a severe motor deficit, with amyotrophy and fasciculation on distal muscles of both lower limbs. Knee reflexes were weak and ankle reflexes were absent. Plantar responses were flexor and cutaneous abdominal reflexes were normal. Sensation, sweating, and sphincter functions were preserved. Serial samples of cerebrospinal fluid showed only a raised protein content at about $80 \mathrm{mg} / \mathrm{dl}$. Electrophoresis was normal. Antibody titers for poliovirus were negative. Motor and sensory conduction velocities and somatosensory-evoked potentials were normal. Electromyograms of tibialis anterior, quadriceps femoris, and semimembranous muscles bilaterally showed fibrillations, fasciculations, and positive potentials. The motor unit potentials had neurogenic characteristics. A myelogram done with metrizamide followed by a computed tomographic scan showed no intradural, extradural, or retroperitoneal abnormalities. Biopsies of the sural nerve and gastrocnemius muscle were obtained for light and electron microscopy examination. The sural nerve was normal. The gastrocnemius muscle, however, exhibited atrophy of a great number of fasciculi, numerous angular fibers with inversion of the enzymatic pattern, and fiber-type grouping (Figure). The neurological symptoms and signs of the patient remain stable as of the time of this report.

The evident cause of the neurological syndrome in this patient was irradiation of the epiconus and conus medullaris of the spinal cord. The total dosage received by these spinal cord segments, resulting from the superimposition of the radiation fields, was estimated at about 8,000 rads, exceeding the maximum tolerable dosage for the spinal cord usually allowed [5]. The clinical picture of the patient was characterized by slowly progressive and self-limited motor deficit and amyotrophy of the lower limbs, which occurred many months after radiotherapy. This syndrome is identical to that described by Greenfield and Stark [1]. The absence of a sensory, sphincter, or sweating disorder makes a lesion of the cauda equina and/or lumbosacral plexi unlikely. The histopathological findings in the muscle biopsy, together with the undamaged sural nerve, are typical of a chronic lesion of the anterior horn. This combination of data suggests a selective 